\title{
Écochampion, écopreneur et écoleader : trois rôles du dirigeant de PME dans le développement des écoinnovations
}

\author{
Kadia Georges $\mathrm{Aka}^{1}$, DBA \\ Laboratoire de recherche sur le Développement Durable et la PME \\ Institut de Recherche sur les PME
}

\section{INTRODUCTION}

Fournir à d'autres dirigeants qui souhaitent développer des écoinnovations, des exemples et des outils pouvant servir d'entraînements.

L'écoinnovation se distingue d'une innovation classique par l'importance de ses impacts environnementaux et sociaux positifs. Par exemple, une auto électrique peut être considérée comme une écoinnovation en comparaison à une auto fonctionnant au pétrole. La première génère des impacts environnementaux moindres alors que son utilité et son apport économique peuvent être semblables à la seconde.

Les dirigeants de PME voient de plus en plus les écoinnovations comme une source d'avantage concurrentiel en raison de leurs aspects environnementaux et sociaux. Cependant, il semble que ces derniers ne savent pas comment les « fabriquer ». Pourtant des « recettes » existent dont les outils d'analyse du cycle de vie des produits (ACV) et d'écoconception. L'ACV est un processus d'analyse visant à faire l'évaluation des impacts environnementaux d'un produit alors que l'écoconception est un processus de conception d'un nouveau produit respectueux de l'environnement.

Bien qu'utiles, ces outils ont montré leurs limites notamment parce qu'ils se focalisent sur les aspects techniques du développement des écoinnovations (p. ex. : quantification des intrants et extrants pour
l'ACV, check-list des enjeux environnementaux pour l'écoconception). De plus, ils sont perçus par les dirigeants de PME comme étant onéreux (p. ex.: coûts d'implantation, coûts d'intervention de consultants externes) et inadaptés à leur mode d'opération (p. ex. : formalisation de certaines méthodes). Il n'est donc pas étonnant que ces derniers n'aient quasiment pas recourt à ces outils et continuent de manifester le besoin de comprendre et d'apprendre comment développer des écoinnovations ${ }^{2}$.

Ce qui est au centre des préoccupations des dirigeants de PME, bien plus que les aspects techniques, ce sont plutôt « les manières », « les façons de faire », autrement dit leurs rôles dans le développement des écoinnovations. Dans la mesure où les interactions sociales et les relations de collaboration constituent des façons de faire $\mathrm{qu}^{\prime}$ «affectionnent » les dirigeants de PME en matière d'innovation, c'est en s'intéressant à cellesci que nous pouvons avoir une meilleure compréhension de ce en quoi consistent leurs rôles dans le développement des écoinnovations.

À partir de l'expérience vécue par des dirigeants de PME parvenus à développer des écoinnovations, il est alors intéressant de savoir comment ceux-ci s'y sont pris. Le but : fournir à d'autres dirigeants qui souhaitent développer des écoinnovations, des exemples et des outils pouvant servir d'entraînements.

\section{LES RÔLES DU DIRIGEANT DE PME,... PRESQU'AU QUOTIDIEN}

Les études sur les rôles du dirigeant de PME montrent que ceux-ci varient selon la taille de l'entreprise, le secteur d'activités, le degré d'incertitude du contexte, la structure de propriété. Bref, le dirigeant de PME change constamment de rôles $^{3}$ (p. ex. : symboles, agent de liaison, leader, observateur actif, diffuseur, porte-parole, entrepreneur, régulateur répartiteur de ressources, négociateur) compte tenu du contexte interne et externe de l'entreprise. Mais au quotidien, il est 
confronté à l'information, la décision et les relations interpersonnelles dans l'exercice de ses rôles. Il s'appuie sur ces trois éléments pour conduire ses activités stratégiques, c'est-à-dire celles qui comportent des enjeux pour la compétitivité ou la survie de son entreprise (voir figure 1).

\section{Figure 1 - Trois rôles importants du dirigeant de PME dans la conduite de ses activités stratégiques}

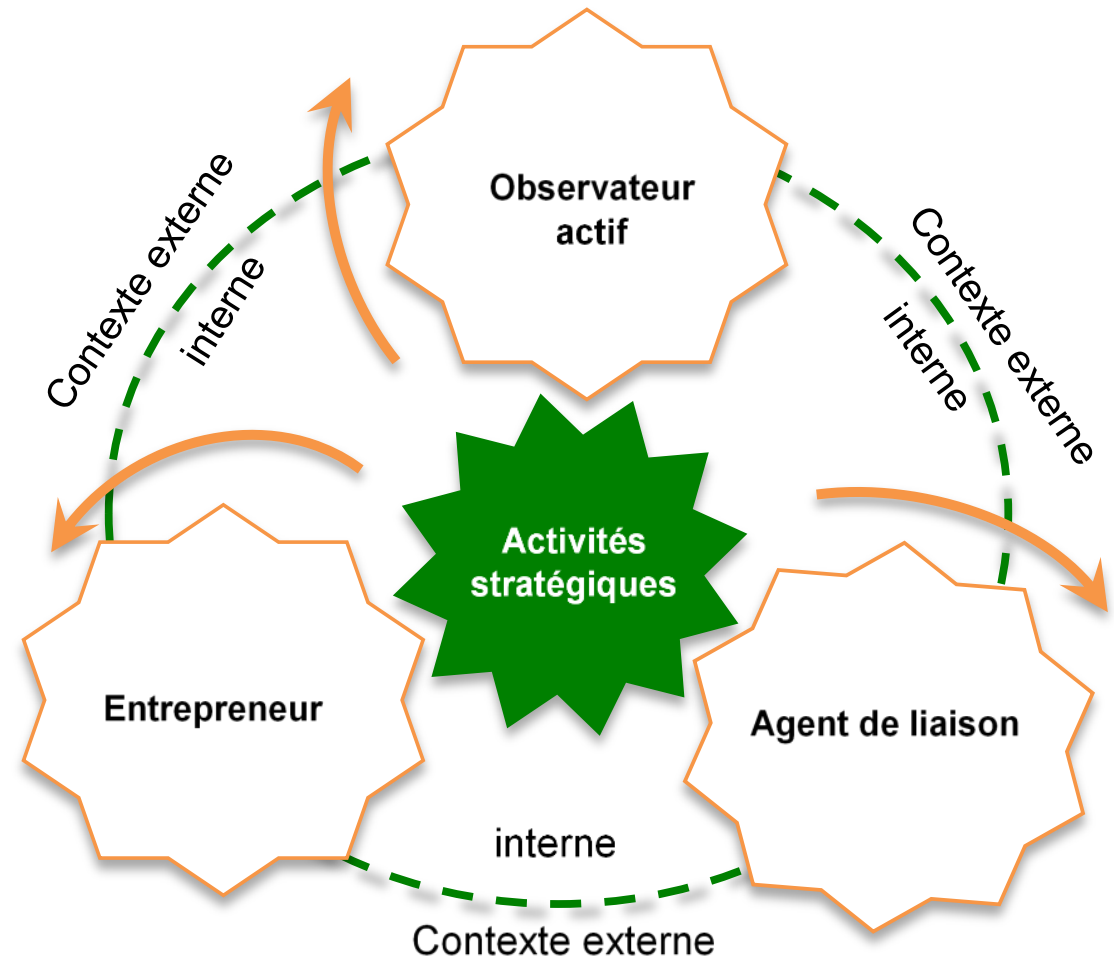

Ainsi, c'est en tant qu'observateur actif de son milieu (l'entreprise et son contexte externe) que celui-ci cherche des informations particulières, identifie les enjeux stratégiques qu'elles renferment et perçoit les intérêts et les préoccupations des acteurs touchés par ces enjeux. Ce rôle est central tant il sert de point de départ à la prise de décision.

Dans son rôle d'entrepreneur, c'est d'abord le dirigeant de PME qui prend la décision d'opérer les changements individuels, structurels et opérationnels à l'intérieur de son entreprise afin de résoudre les problèmes qu'il perçoit. Il initie toute action permettant d'exploiter les opportunités qui se présentent à lui et de les transformer en innovation. Mais, ce rôle lui donne aussi l'occasion de se tourner vers des partenaires externes à qui il doit «vendre » son projet d'innovation.
C'est comme agent de liaison que le dirigeant de PME lie son entreprise avec des partenaires externes qui l'aideront à concrétiser opportunités et projets d'innovation. Dans ce rôle, le dirigeant de PME établit des contacts, entretient des relations, collabore avec les partenaires pour échanger des informations et des ressources.

\section{C'est comme agent de liaison que le} dirigeant de PME lie son entreprise avec des partenaires externes qui l'aideront à concrétiser opportunités et projets d'innovation.

Le dirigeant de PME atteint ses objectifs en effectuant ces trois principaux rôles de façon récursive, par des allers et retours ainsi que des essais et erreurs. Selon la situation et la nature de l'action dans laquelle il s'engage, le dirigeant de PME met, à des degrés divers, l'accent sur chacun de ces rôles. 


\section{LES RÔLES DU DIRIGEANT DE PME ET LE DÉVELOPPEMENT DES ÉCOINNOVATIONS}

Les rôles d'observateur actif, d'entrepreneur et d'agent de liaison sont pertinents dans le développement des écoinnovations. Selon la littérature à ce propos, le développement des écoinnovations est une activité stratégique compte tenu des enjeux environnementaux et sociaux auxquels font face les PME. Il nécessite l'implication d'une diversité de partenaires ayant des intérêts économiques, environnementaux, sociaux différents voire contradictoires. La gestion de ces partenaires et de leurs intérêts oblige le dirigeant à aller au-delà de son rôle de manager «classique ». $\mathrm{Ce}$ dernier doit agir comme un champion du développement durable (écochampion), un entrepreneur durable (écopreneur) ou un leader environnemental (écoleader); ce qui correspond respectivement aux rôles d'observateur actif, d'entrepreneur et d'agent de liaison (voir tableau 1).

Tableau 1 - Les trois principaux rôles du dirigeant dans une démarche d'écoinnovation

\begin{tabular}{|c|c|}
\hline Écochampion & Observateur actif \\
\hline \multicolumn{2}{|c|}{ Axé sur l'information } \\
\hline $\begin{array}{l}\text { - Percevoir des enjeux environnementaux et sociaux. } \\
\text { - Interpréter les informations liées à ces enjeux. } \\
\text { - Promouvoir les enjeux environnementaux et sociaux. }\end{array}$ & $\begin{array}{l}\text { Il est en quête d'informations afin de détecter les changements, } \\
\text { d'identifier les problèmes et les opportunités, d'accumuler des } \\
\text { données sur le milieu. Il utilise un certain nombre de moyens pour } \\
\text { acquérir une meilleure compréhension des tendances de son } \\
\text { environnement et pour s'informer des idées nouvelles. }\end{array}$ \\
\hline Écopreneur & Entrepreneur \\
\hline \multicolumn{2}{|c|}{ Axé sur la décision } \\
\hline $\begin{array}{l}\text { - Saisir une opportunité environnementale et sociale. } \\
\text { - Définir un projet d'écoinnovation à partir de cette opportunité. } \\
\text { - Mobiliser des ressources afin de réaliser l'écoinnovation. }\end{array}$ & $\begin{array}{l}\text { Il cherche à détecter de nouvelles opportunités et de nouveaux } \\
\text { problèmes, et prend l'initiative de projets d'amélioration pour en } \\
\text { tirer parti ou y faire face. Lorsqu'il a découvert un problème ou } \\
\text { une opportunité, il peut décider que son organisation doit agir pour } \\
\text { améliorer une situation donnée. Il tient compte des intérêts des } \\
\text { acteurs pouvant lui apporter des ressources }\end{array}$ \\
\hline Écoleader & Agent de liaison \\
\hline \multicolumn{2}{|c|}{ Axé sur les relations interpersonnelles } \\
\hline $\begin{array}{l}\text { - Mobiliser des partenaires externes. } \\
\text { - Constituer un réseau de partenaires-clés. } \\
\text { - Acquérir et échanger des ressources. }\end{array}$ & $\begin{array}{l}\text { Il établit son réseau de contacts externes; [...] il s'en sert pour } \\
\text { obtenir des ressources. Il prend les choses en mains lorsque son } \\
\text { organisation doit conduire des négociations importantes avec des } \\
\text { acteurs externes. }\end{array}$ \\
\hline
\end{tabular}

Nous voulions ainsi comprendre à travers ces histoires, en quoi consistent les trois principaux rôles (écochampion, écopreneur, écoleader) et leurs particularités.

Le champion du développement durable ou l'écochampion est celui qui scanne constamment son milieu pour y sélectionner des informations liées à des enjeux environnementaux et sociaux (p. ex.: changement climatique, santé et sécurité des produits, pollution atmosphérique, agitation sociale) pouvant avoir des incidences sur ses activités. L'écopreneur a pour rôle de détecter les opportunités environnementales et sociales que renferment ces enjeux et de les transformer en écoinnovation. L'écoleader est celui qui parvient à concilier et à intégrer les demandes, les préoccupations et les intérêts environnementaux et sociaux des partenaires dans les caractéristiques de l'écoinnovation.
Pour ce faire, il doit être capable de les influencer et de les mobiliser autour du projet d'écoinnovation afin de bénéficier de leurs ressources.

C'est ce que nous avons observé sur le terrain avec plusieurs dirigeants ${ }^{4}$ de PME de tailles différentes qui sont parvenus à développer des écoinnovations, soit des technologies propres, des produits écologiques et équitables, qui toutes répondent à des enjeux environnementaux et sociaux différents dans des secteurs d'activités variés (p.ex.: traitement des eaux industrielles, meuble, vélo, cosmétique, valorisation des déchets). Nous avons demandé à ces dirigeants de nous raconter dans les détails l'histoire du développement de leurs écoinnovations. Nous voulions ainsi comprendre à travers ces histoires, en quoi consistent les trois principaux rôles (écochampion, écopreneur, écoleader) et leurs particularités lorsque les dirigeants de PME sont engagés dans une démarche d'écoinnovation. 


\section{L'ÉCOCHAMPION... UNE STRATÉGIE}

En tant qu'observateurs actifs ou écochampions, nos dirigeants de PME se comportent comme des stratèges. Ils perçoivent tout d'abord un enjeu environnemental, social et saisissent les opportunités d'écoinnovations qu'il comporte. Ensuite, ils définissent un projet d'écoinnovation et se dotent enfin des moyens pour les réaliser dans un espace qu'ils délimitent. Nous avons clairement noté cette action stratégique des dirigeants dans leur rôle d'écochampion.

Par exemple, le dirigeant de PME à l'origine d'un vélo électrique hybride n'est pas le seul, dans son secteur d'activité, à voir l'électrification du vélo comme une information liée à un enjeu de réduction des gaz à effet de serre par l'utilisation d'une énergie renouvelable moins polluante (soit l'hydroélectricité). D'autres entreprises concurrentes ont certainement vu le fait de se transporter sans pétrole et sans auto comme une information, une opportunité. Cependant, en tant qu'écochampion, c'est le dirigeant qui perçoit cette information

\section{L'ÉCOPRENEUR... UN TACTICIEN}

En tant qu'entrepreneurs ou écopreneurs, nos dirigeants de PME se comportent comme des tacticiens. Ils se mettent dans la peau d'un client, un utilisateur, un évaluateur de ce qui deviendra plus tard une écoinnovation, ou de tout acteur qui contribue par ses ressources à la conception et à la concrétisation de l'écoinnovation. Pour ce faire, ils s'engagent dans des expérimentations collectives qui permettent de détecter, de saisir des opportunités environnementales et sociales.

Par exemple, le dirigeant à l'initiative d'un meuble en carton recyclé, nous confiait ceci : « En partant de mes valeurs, j'ai cherché sur Google les mots : écoresponsable, ludique, pratique, original et meuble. À la première page, les résultats des

\section{L'ÉCOLEADER... UN RÉSEAUTEUR}

En tant qu'agents de liaison ou écoleaders, nos dirigeants de PME agissent comme des réseauteurs qui, en plus de mobiliser et d'intéresser des partenaires externes au projet d'écoinnovation, permet- comme pouvant avoir une incidence sur ses activités pendant que les autres «ne le font pas ».

La particularité du stratège dans son rôle d'écochampion est en fait la victoire du lieu sur le temps qui permet au dirigeant de PME de capitaliser des avantages acquis (p. ex.: présence de l'hydroélectricité au Québec, réputation nationale dans la fabrication de vélos, savoir-faire technologique), de préparer les expansions futures et de se donner ainsi une indépendance par rapport à la variabilité des circonstances (p. ex.: évolution des habitudes d'utilisation du vélo, sensibilité des consommateurs aux produits respectueux de l'environnement). Par la suite, il est plus aisé pour lui de saisir l'opportunité environnementale et sociale qui lui permet de mieux exploiter ses avantages.

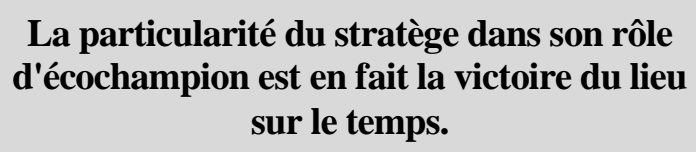

recherches mentionnent le métier de "cartonniste". Celui-ci est bien développé en France. Mais au Québec, pays de la pâte à papier, les entreprises commencent à réutiliser le carton. J'ai donc vu l'occasion de valoriser le carton en concevant et en commercialisant des meubles en carton recyclé ».

En particulier, dans son rôle d'écopreneur le tacticien n'a pour lieu d'action que celui de l'autre. Il doit jouer avec un terrain qui n'est pas le sien et qui lui est souvent imposé. Dans ces conditions, le rôle du dirigeant de PME se résume à l'art de «faire des coups », le sens de l'occasion. Par la suite, le tacticien est celui qui collabore avec des partenaires afin de concrétiser son écoinnovation. Pour ce faire, il va agir comme un écoleader.

tent de créer et de maintenir un réseau restreint de partenaires-clés qui donneront à l'écoinnovation ses caractéristiques définitives. À propos des partenaires-clés, ce sont des acteurs actifs d'un 
projet qui les intéresse et qui ne peut fonctionner sans eux, parce qu'ils possèdent les ressources cruciales pour sa concrétisation. Il s'agit alors pour l'écoleader d'affecter «les bons acteurs aux bons rôles ».

Pour créer le réseau de partenaires, nos dirigeants doivent réconcilier les différents intérêts des partenaires qu'ils mobilisent en faisant de nouvelles combinaisons ou associations $\mathrm{d}$ '« ingrédients » appartenant à des «traditions » différentes. Par exemple, la combinaison entre le carton recyclé et le meuble donne le meuble en carton recyclé qui tout en étant écologique répond aux critères de qualité des clients. Idem pour l'association de la propulsion mécanique à la propulsion électrique qui conduit au vélo électrique hybride qui, tout en contribuant à la réduction des gaz à effet de serre, comble les besoins de santé et de sécurité des personnes âgées et des jeunes travailleurs, et respecte les standards des agences de règlementation du transport.

Afin de maintenir ce réseau, il intéresse les partenaires en définissant avec eux les caractéristiques de base communes de l'écoinnovation qui rejoignent leurs intérêts à des degrés divers. Par exemple, une matière naturelle, un matériau recyclé, un composant non toxique, une règlementation environnementale, un équipement technique particulier sont autant de dispositifs matériels ou immatériels obligatoires que tous les acteurs utilisent quels que soient leurs usages individuels de l'écoinnovation et qui en constituent les caractéristiques de base communes. En fait, le réseauteur dans son rôle d'écoleader cherche à légitimer l'écoinnovation afin de stabiliser ses caractéristiques et de rendre irreversible sa concrétisation.

\section{EN CONCLUSION... DES ENCHAÎNEMENTS DE RÔLES INTERRELIÉS ET INTERDÉPENDANTS}

Dans notre étude, en « suivant à la trace » des dirigeants de PME dans le développement des écoinnovations, nous sommes parvenus à proposer une perspective cohérente d'ensemble de leurs rôles. Cette perspective donne, au moins en partie, une vision réaliste et intégrée de ce que les dirigeants de PME peuvent faire afin de développer des écoinnovations. Cette vision se résume à des enchaînements de rôles interreliés et interdépendants (voir figure 2).

\section{Figure 2 - Processus des rôles du dirigeant de PME dans le développement des écoinnovations}

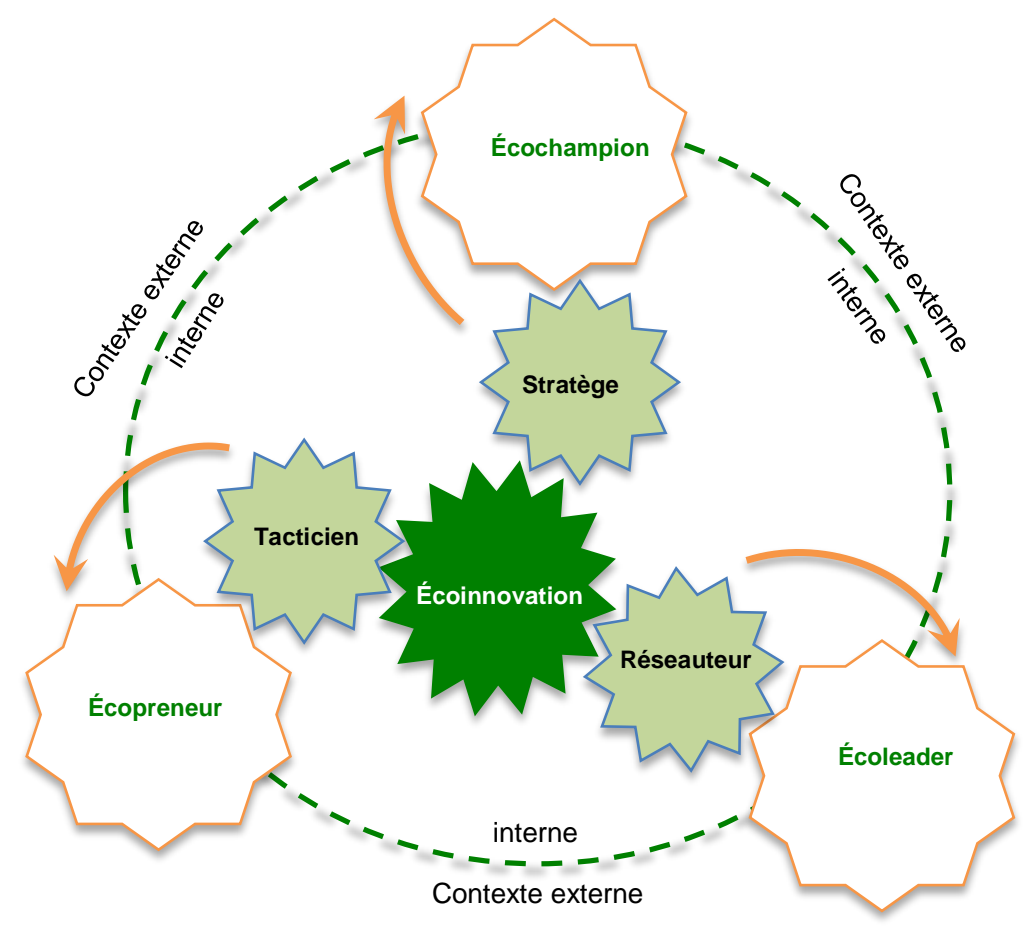




\section{Les entrepreneurs et dirigeants de PME peuvent acquérir, renforcer leurs compétences dans l'un ou l'autre des trois principaux rôles ou identifier parmi leurs collaborateurs internes ceux qui vont mieux les exercer.}

Les dirigeants de PME peuvent donc agir comme des écochampions, des écopreneurs et des écoleaders lorsqu'ils souhaitent s'engager dans une démarche d'écoinnovation. Mais, mieux encore, ils doivent mettre l'accent sur les rôles de stratège, de tacticien et de réseauteur qui, dans le contexte des écoinnovations et des PME de notre étude, se sont avérés bénéfiques à la fois pour « démarrer » le processus que pour l'« huiler ».

Dans la littérature en management ${ }^{5}$ et beaucoup moins en gestion des écoinnovations, ces rôles sont peu étudiés et méritent d'être approfondis. Les résultats de notre étude s'adressent aussi aux entrepreneurs et dirigeants de PME ainsi qu'aux organismes qui les soutiennent dans leur projet d'écoinnovation.

Les entrepreneurs et dirigeants de PME peuvent acquérir, renforcer leurs compétences dans l'un ou l'autre des trois principaux rôles ou identifier parmi leurs collaborateurs internes ceux qui vont mieux les exercer, lorsqu'ils s'engagent dans une démarche d'écoinnovation. L'objectif : amplifier les chances de succès de leurs écoinnovations en confiant « les bons rôles aux bonnes personnes ».

Pour contribuer à l'atteinte de cet objectif, les organismes peuvent introduire dans leur programme d'appui aux entrepreneurs et dirigeants de PME, le renforcement de leurs capacités d'écochampion, d'écopreneur et d'écoleader ainsi que de tacticien, de stratège et de réseauteur. Par ailleurs, ils peuvent orienter leurs efforts sur les processus sociaux et interactifs (p. ex. : mapping et développement des relations de collaboration externes, constitution de réseaux, choix des partenaires-clés, intermédiation) du développement des écoinnovations.

\section{BIBLIOGRAPHIE}

Aka, K. G. (2015). Que font les dirigeants de PME pour développer des innovations durables (écoinnovations)? Thèse de doctorat, Université du Québec à Trois-Rivières, Trois-Rivières, Québec, Canada.

Florén, H. et Tell, J. (2004). What Do OwnersManagers in Small Firms Really Do? Differences in Managerial Behavior in Small and Large Organizations. Small Enterprise Research, 12(1), 5770.

Hall, J et Vredenburg, H. (2003). The Challenges of Innovating for Sustainable Development. MIT Sloan Management Review, 45(1), 61-68.

Mintzberg, H. (2009). Managing. Berrett-Koehler Publishers, Inc., San Francisco, Californie.

Réseau Entreprise et Dévelopement durable (2014). Simplifying complexity. The 8 sustainability challenges for Canadian business 2014. NBS Report. Disponible sur http://nbs.net/wpcontent/uploads/NBS-Challenges-2014.pdf

Tengblad, S. (2006). Is there a 'New Managerial Work'? A Comparison with Henry Mintzberg's Classic Study 30 Years Later. Journal of Management Studies 43(7), 1437-1461.

\section{NOTES}

${ }^{1}$ Le contenu de ce bulletin s'appuie sur la thèse de doctorat réalisée par Aka, K. G. sous la direction de Trépanier, M. et Labelle, F.

${ }^{2}$ Voir les rapports 2012, 2013 et 2014 du Réseau Entreprise et Développement Durable (REDD).

${ }^{3}$ Voir entre autres les travaux de Florén et Tell (2012) qui confirment ces rôles identifiés par Mintzberg (2009), mais dans le contexte des PME.

${ }^{4}$ Pour une description détaillée de ces PME (présentation des PME, leurs écoinnovations et récits historiques), le lecteur est invitié à consulter la thèse de l'auteur.

${ }^{5}$ Pour plus de précisions à ce propos, le lecteur peut se référer aux travaux de Tengblad (2006). 